\title{
Suppression Methods for Electromagnetic Interference of the DC Motor
}

$$
\text { Qun } \mathrm{Hou}^{1, \mathrm{a}} \text {, Yifan Zhou }{ }^{2, \mathrm{~b}} \text {, and Xin Peng }{ }^{3, \mathrm{c}} \text {, Long Zhang }{ }^{3, \mathrm{c}}
$$

School of Physics and Information Engineering, Jianghan University, Wuhan City, Hubei

Province, China

aviphouqun@163.com, ${ }^{b} 2324174340 @ q q . c o m,{ }^{c} 307301544 @ q q . c o m,{ }^{c} 785608724$

@qq.com

Keywords: DC motor, Electromagnetic Interference, Filter, Suppression Measures,
Electromagnetic Compatibility

Abstract. The basic principle of electromagnetic interference, test methods, and suppression measures for DC motor are studied and analyzed. We select 18V/5A DC motor as the research object, and test full-frequency interference characteristics of the conducted and radiated interference. By analyzing the test data, we design and add the X2Y filter to the DC motor, and test its all-frequency characteristics. The results show that the X2Y filter can effectively suppress the conducted and radiated electromagnetic interference of DC motor, which can greatly enhance the electromagnetic compatibility characteristics of the DC motor.

\section{Introduction}

At present, the people's living environment has been surrounded by electromagnetic environment, at the same time, with the rapid growth of population, a large number of electronic, and information equipment have come into people's lives. Many electronic components are incorporated on these equipments, which would be interacted with each other by electromagnetic radiations and conducted signal, making the electromagnetic environment worse; meanwhile, it also poses a threat to the communication, computer and electronic system.

Electromagnetic Compatibility (EMC), it refers to the coexistence state of the electrical and electronic equipment that can perform their functions in a common electromagnetic environment ${ }^{[1]}$, which requires that these various devices in the same electromagnetic environment can work without disturbing each other and achieve "compliant" status.

Because the DC motors are widely used in household appliances and electrical equipments, it is important to study the electromagnetic compatibility of the DC motors. In this article, we select $18 \mathrm{~V} / 5 \mathrm{~A}$ DC motor as the research object, and its test EMI characteristics exceed the standard limit. Therefore, we design and add the X2Y filter to the DC motor. The results show that the X2Y filter ${ }^{[2]}$ can significantly reduce the level of the electromagnetic interference ${ }^{[3]}$ and enhance the electromagnetic compatibility of the DC motor.

\section{The basic principle of electromagnetic interference}

EMI (Electromagnetic Interference) is "Electromagnetic disturbance caused by device, transmission channel or system performance decline". In the cable harness, multi-conductor transmission line, and in close proximity of the wires of the printed circuit board, there is a variety of electromagnetic interference, and the running motor also will produce strong electromagnetic interference, causing worsening phenomenon of the device or system. Moreover, it can interfere with the normal operation of other equipment, which can lead to disastrous chemical accidents. So the study of the motor electromagnetic compatibility problem is particularly important.

\subsection{The three elements of EMI}

No matter complex system or simple equipment, any major scientific content of electromagnetic compatibility all revolve around three elements: electromagnetic interference source, route of transmission and sensitive equipment. As shown in Fig.1, interference sources are accompanied 
with sensitive equipment, and there may be party of the potential interference path from one side to the other, which it the transmission route. The interference transmission path is divided into two kinds, radiation interference and conduction interference ${ }^{[4]}$. Radiation interference mainly focuses on disturbance electromagnetic waves under the far field; the main study of conduction interference is the effect the distribution parameters of the transmission line and the current transmission mode on noise transmission.

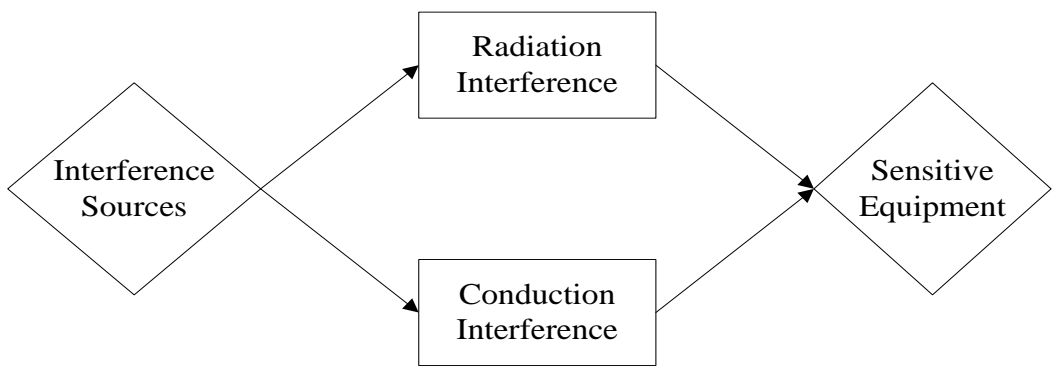

Fig.1 The three elements of EMI and their relationships

\subsection{The principle of motor EMI}

The DC motor with commutator and brushes belongs to the inductive load. When the motor is working, suddenly cut off the electric power, the coil winding will produce an inductive load impact. The brush transmits from one side to the other side, because he contact resistance dramatic changes, resulting in mutation of the current, and therefore to produce electric arc, which will produce a strong electromagnetic interference between the brush and the rotor. The electromagnetic interference frequency is about $0.15 \sim 300 \mathrm{MHz}^{[5]}$, which has a wide spectrum, rich harmonic and other characteristics.

In order to further study the generation mechanism of electromagnetic interference, we specially choose a DC18V/5A motor, which has not carried out test of electromagnetic compatibility. The experiment of electromagnetic radiation is carried out in the Engineering Technology Research Center, which is according to demand of test, with $1 \mathrm{~m}$ away from the receiving antenna ${ }^{[6]}$.

\section{The test results of electromagnetic interference of DC motor}

\subsection{The conducted interference voltage test}

Experimental measurement frequency range is $0.15 \sim 30 \mathrm{MHz}$, using Japanese U3571 spectrum analyzer and LS1-15V $50 \Omega / 50 \mu \mathrm{H}$ artificial mains network to make voltage measurement of conducted interference of the motor. The standard limit of the conducted interference voltage of the EN55014 is $0.15 \sim 30 \mathrm{MHz}$, and quasi-peak is $66 \sim 56 d B \mu V^{[7]}$. It is showed in Fig.2 (a) that testing peak values of conducted interference voltage are made by $18 \mathrm{~V}$ DC motor in open state. Maximum conducted EMI of this motor without EMI filter is about $73 d B \mu V$. Compared with the specified limit of conducted interference voltage, it exceeds $10 \mathrm{~dB} \mu V$ within the range of $0.15 \sim 0.5 \mathrm{MHz}$.

\subsection{The radiation interference voltage test}

Experimental measurement frequency range is $30 \sim 300 \mathrm{MHz}$. The field is the GTEM room, and a spectrum analyzer is used to test. In Fig.2 (b), it is the result of the testing peak values of radiation interference voltage when the $18 \mathrm{~V} / 5 \mathrm{~A}$ DC motor is in open state. The standard limit of EN55014 radiation interference power of is $18 \sim 20 \mathrm{dBpW}$. The testing results show that without EMI filter, maximum radiation interference of the motor is about $60 \mathrm{~dB} \mu \mathrm{V}$, of which frequency band have exceed the limits of radiated interference voltage in $30 \sim 300 \mathrm{MHz}$. 


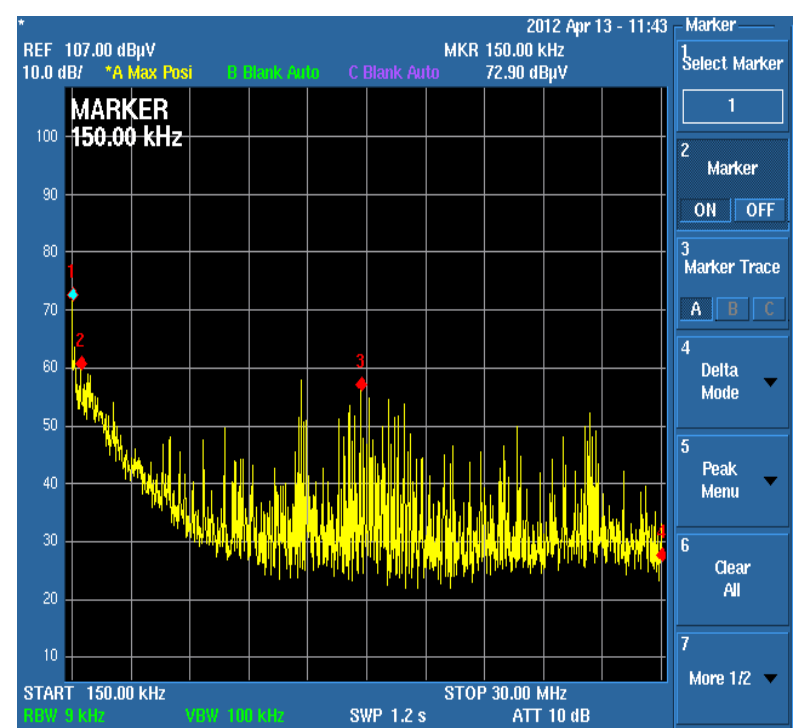

(a)

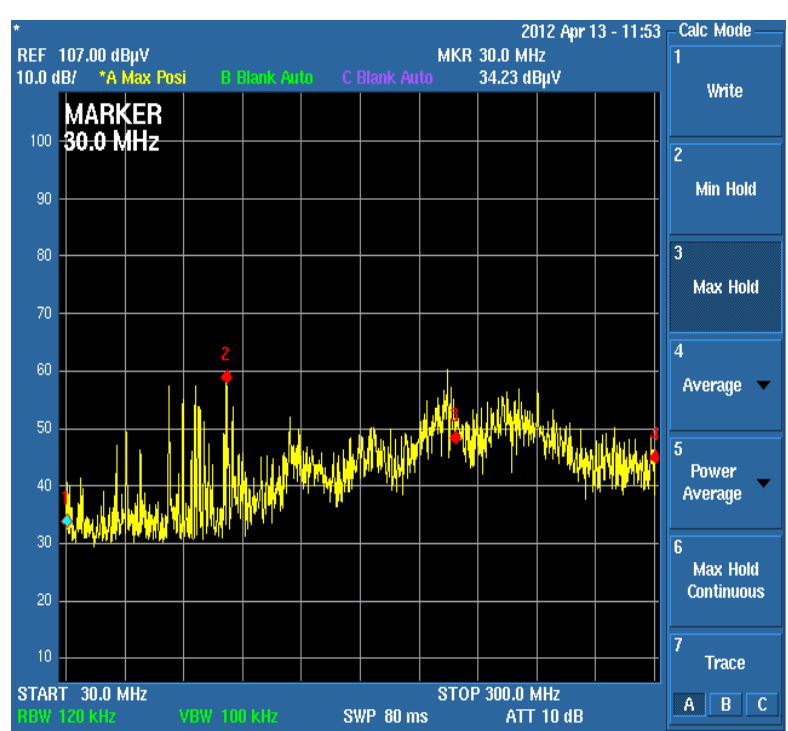

(b)

Fig.2 (a) and (b) respectively show noise amplitude frequency characteristics of 18V/5A DC motor in $0.15 \sim 30 \mathrm{MHz}$ and 30 300MHz without EMI filter

\section{Suppression measures of DC motor}

Although it is difficult to eliminate EMI, we can take a variety of measures to restrain EMI, to reduce the influence on the system or equipment. During the EMC design, the filter is quite critical and complex technology, and the filter is the main protection measures of conduction interference and radiation interference. Fig.3 (a) and 3(b) show the voltage test results of conducted interference and radiated interference after adding X2Y filter circuit to $18 \mathrm{~V}$ DC motor. Compared with those of Fig.2 (a) with Fig.3 (a), the conducted interference reduces by $25 d B \mu V$ in $0.15 \sim 0.5 \mathrm{MHz}$, and the radiation interference reduces by $40 \mathrm{~dB} \mu \mathrm{V}$ in $50 \sim 100 \mathrm{MHz}$, whose interference characteristics have satisfied the limits of EN55014 standard.

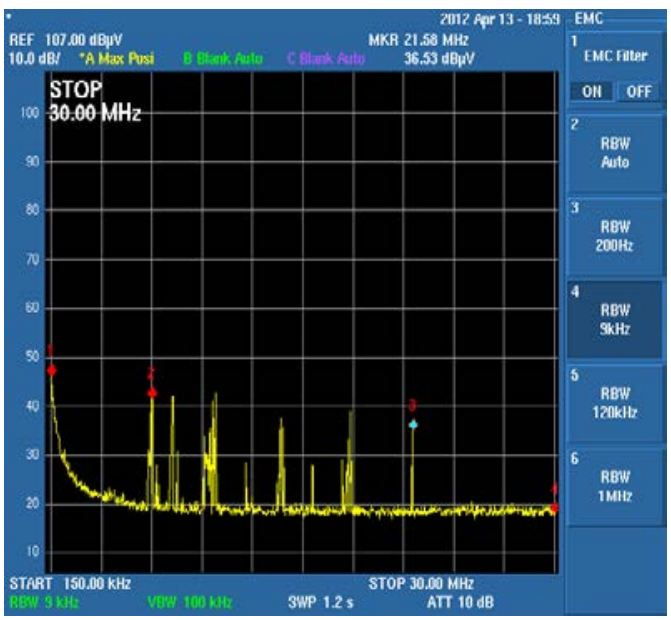

(a)

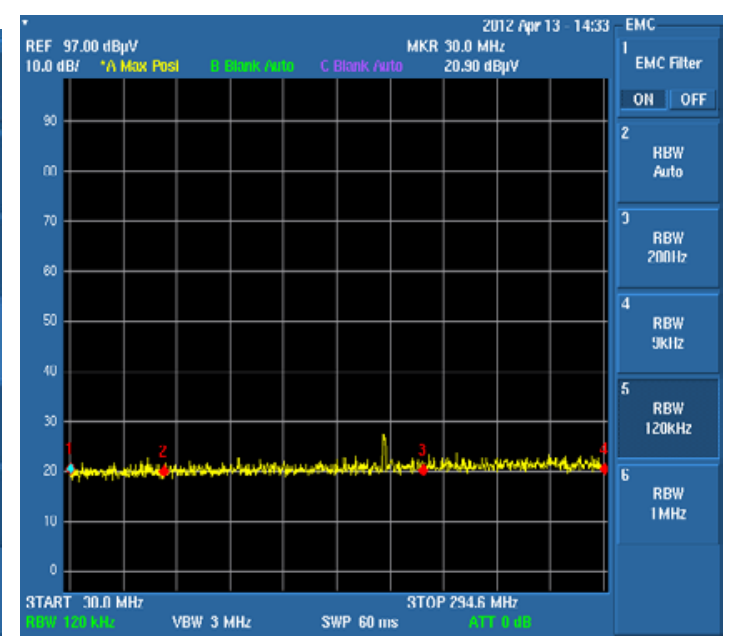

(b)

Fig.3 (a) and (b) respectively show noise amplitude frequency characteristics of 18V/5A DC motor in $0.15 \sim 30 \mathrm{MHz}$ and 30 300MHz with EMI filter

\section{Conclusion}

In this article we analysis the basic principle of the motor EMI, and take 18V/5A DC motor as the research object. Its frequency characteristics of conducted interference and radiated interference are measured, which show that their mean interference value exceeds the specified limit. Based on data parameters, we design and add the X2Y filter circuit to the motor, and test the amplitude frequency characteristics. The results show that the X2Y filter circuit can better restrain the 
interference characteristics of the motor. It completely satisfies the limits of radiation intensity in EN55014 standard, and greatly enhances electromagnetic compatibility characteristic of the DC motor.

\section{References}

[1] Yinjing Guo, Wenhong lv, Fuhua Tang.et al. In Chinese: Principles and Applications of Electromagnetic Compatibility [M]. Beijing: Tsinghua University Press, (2004), p.129-131.

[2] Janchang Ou. X2Y filter is a new kind of EMI filter [J]. Electrical Engineering, 2004(10)

[3] S.Chen, T.W.Nel, J.S.Lai, et al. Toward EMI Prediction of a PM Motor Driver for Automotive Applications [J]. IEEE Applied Power electronics Conf, 2003(1): 14-22.

[4] Zhengguang Liang, Renyuan Tang. Problems of electromagnetic compatibility for electric machines [J]. S\&M Electric Machines 2004.31(2):63-67.

[5] Kuos M, Morgan D R, Active noise control a tutorial review [J]. Proceedings of the IEEE, 1999, 87(6):943-973.

[6] Wei Wu. Low-voltage Electrical Products and Detect EMC Standards [J] Low-voltage Electrical,2004 (9)

[7] Jinglin Liu. Electromagnetic compatibility requirements of electrical products and standards [J] Electrical Technology Magazine, 2001,(11): 24 26. 